\title{
Importancia de los Sistemas de Documentación en el Manejo de los Recursos Fitogenéticos ${ }^{1}$
}

\author{
Froylan Rincón $S .{ }^{2}$, Luis G. González R. ${ }^{3}$
}

\section{INTRODUCCIÓN}

La diversidad genética de plantas útiles y sus parientes sitvestfes que ha sido recolectada, es depositada en bancos de germoplasma, y de esta manera se encuentra a disposición del personal técnico/científico que lo desee.

Para que dicho germoplasma sea usado eficientemente por los programas de mejoramiento, debe existir información lo más completa posible sobre las características más importantes que describan a esos materiales, por ejemplo, tipo de planta, viabilidad de semillas, etc. Así los usuarios pueden seleccionar más eficientemente, el germoplasma que necesiten. Sin embargo, uno de los principales problemas en la evaluación y utilización del material genético, así como de la información respectiva, recae en la falta de información sistemática sobre inventarios y descripción confiable del germoplasma.

\section{ACTIVIDADES PRINCIPALES DE UN BANCO DE GERMOPLASMA}

El material genético es introducido al banco de germoplasma de varias maneras, ya sea como resultado de misiones de recolección o bien por el intercambio con otros bancos de germoplasma u otras instituciones. En ambos casos el movimiento de germoplasma, aún dentro del mismo país, debe someterse a las normas o regulaciones fitosanitarias vigentes.

Al incorporarse el material al banco, se le asigna un número de accesión, el cual será el identificador único. Una vez registrado, elmaterial pasa a la fase de acondicionamiento, que en el caso de semillas ortodoxas (e.g. frijol). contempla limpieza, secado, determinación del contenido de humedad, y porcentaje de viabilidad, siguiendo recomendaciones especificas para cada cultivo. El material con altos fndicesde germinación/viabilidad, es empaquetado herméticamente para que no se exponga a posibles cambios ambientales, que modificarían el contenido de humedad en las semillas (con el subsecuente deterioro) y luego es almacenado a bajas temperaturas.

Durante el período de conservación en el banco, se extraen muestras representativas para distribución/intercambio del germoplasma e inspecciones periódicas, que determinaran el material genético que debe ser regenerado/incrementado, en base a tamaño de muestra, contenido de humedad y porcentaje de viabilidad.
Igualmente, el germoplasma que vaa ser almacenado en el campo o in vitro, debe ser acondicionado e inspeccionado regularmente para garantizar su viabilidad y representatividad.

Con la finalidad de que el germoplasma sea aprovechado al máximo, este debe ser caracterizado y evaluado en una forma sistemática, para que de esta manera, el usuario pueda seleccionar más eficientemente los materiales que necesita para su ulterior o inmediata utilización.

\section{PROCESAMIENTO DE LA INFORMACIÓN}

En cada una de las diferentes actividades que se llevan a cabo en un banco de germoplasma y previo a ellas, se genera información de gran utilidad sobre cada accesión, la cual debe estar debidamente documentada, con el objetivo de que tanto el banco como el material genético mismo sean manejados eficientemente. Las actividades principales de un banco de germoplasma se ilustran en la Figura 1.

El Consejo Internacional de Recursos Fitogenéticos (IBPGR) se ha preocupado de que el material genético depositado en los bancos de germoplasma sea utilizado eficientemente, por lo que ha promovido reuniones de expertos por cultivo, quienes han definido descriptores únicos para registrar la información sobre el origen (datos de pasaporte), caracterización y evaluación del material genético. Estos descriptores definen las características más relevantes y la forma aunque estas deben ser registradas, permitiendo además, la selección del germoplasma deseado; por otra parte, el uso de estos descriptores propicia un lenguaje comón de la información y a la vez fomenta el intercambio más eficiente tanto de la información como del material genético entre bancos de germoplasma y usuarios en general.

En la actualidad, los descriptores han sido ampliamente aceptados por parte de los usuarios, lo que aunado al desarrollo de los sistemas de información, permiten cumplir con los objetivos de las redes de información por cultivo, así

\footnotetext{
${ }^{1}$ Trabajo presentado en la XXXVI Reunión Anual del PCCMCA. San Salvador, El Salvador, marzo 1990.

${ }^{2}$ Coordinador Asistente, Oficina del IBPGR para Norteamerica, Centroamérica y El Caribe e/o. CIMMYT, Mexico.

${ }^{3}$ Coordinador, Oficina del IBPGR para Norteamerica, Centroamérica y El Caribe c/o CIMMYT, México.

Publicado en Agronomía Mesoamericana. Vol. 2 (1991).
} 


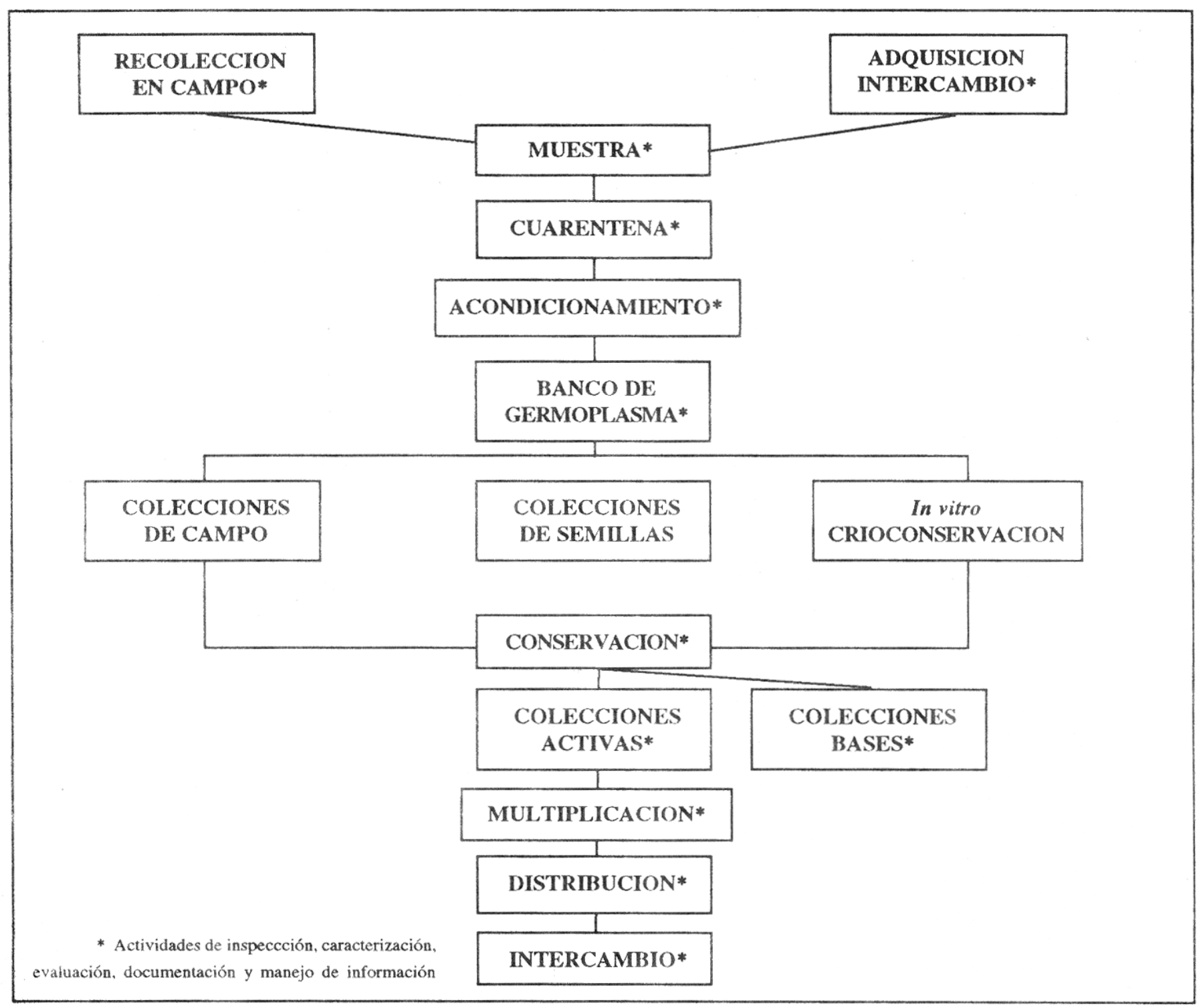

Fgura 1 Actividades principales de un banco de germoplansma.

como del personal involucrado en cada una de las actividades sobre recursos genéticos, Por otra parte, los sistemas computarizados, permiten producir catálogos y directorios de germoplasma, así comoel procesamiento de la información que ierá de utilidad para los mejoradores o usuarios directos del banco de germoplasma.

\section{SISTEMAS DE DOCUMENTACIÓN Y EL MA- NEJO DE LOS RECURSOS FITOGENETICOS}

El uso de sistemas computarizados en programas de recursos genéticos se ha incrementado considerablemente, dada la necesidad de manejar la cantidad de información que se genera en cada una de las actividades desarrolladas en un banco de germoplasma la cual debe ser bien documentada, especialmente cuando se cuenta con gran número de accesiones.
Actualmente existen sistemas de información para el manejo de germoplasma, corno la red de información sobre recursos genéticos (GRIN) la cual fue desarrollada por el Departamento de Agricultura de los Estados Unidos, esta contiene información actualizada y disponible para apoyar el intercambio de material vegetal y su información entre investigadores y fitomejoradores; de la misma manera, en el Banco de Semillas de Kew, U. K., el sistema de documentación permite eliminar la duplicación de esfuerzos en el manejo de la información básica, el control de las accesiones y la distribución de semillas. Siguiendo las recomendaciones del "Taller Mundial sobre Germoplasma de Maíz" realizado en 1988; el CIMMYT (Centro Internacional de Mejoramiento de Maíz y Trigo) y el IBPGR han promovido conjuntamente el establecimiento de una "Base Mundial de Datos sobre Germoplasma de Maíz". Previamente, se produjo un disco compacto que contiene datos pasaporte de 10,533 


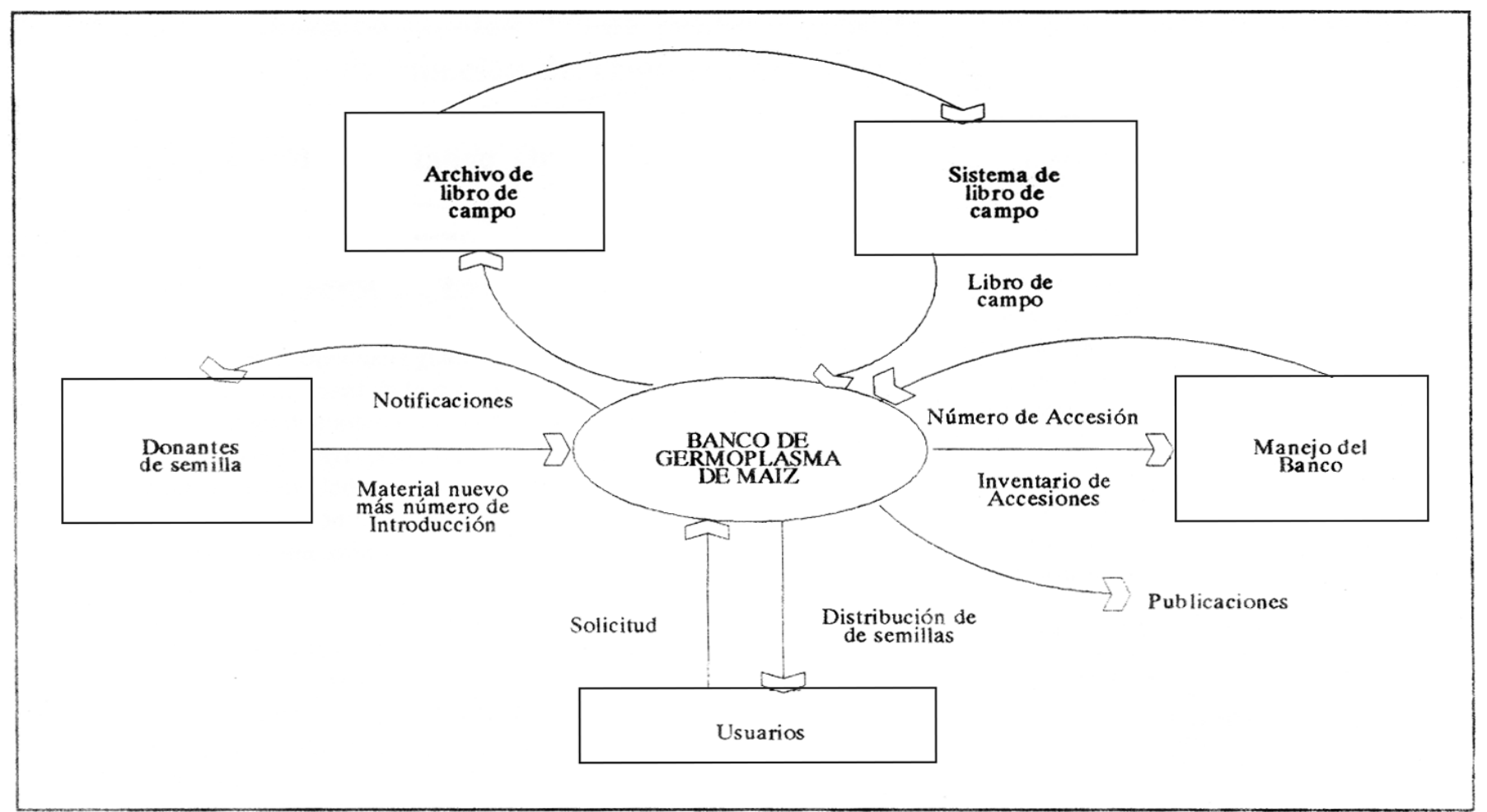

Figura 2 Esquema general de las actividades del Banco de Germoplasma de Maíz del CIMMYT.

accesiones del banco de germoplasma de maíz del CIMMYT, donde se contemplan 29 descriptores para maíz, Se pretende que este sea distribuido a los programas nacionales, promoviéndose de esta manera la utilización del germoplasma y el intercambio de la información respectiva entre programas. En la Figura 2, se representa en forma esquemática las actividades generales del banco de germoplasma de maíz del CIMMYT; de la misma manera, la relación entre estas actividades en detalle, y la generación de la información en cada etapa respectiva (Figura 3), lo que constituye el sistema de manejo de información sobre germoplasma de maíz en el CIMMYT.

Con el propósito de integrar a recolectores de material genético, jefes de bancos de germoplasma, y usuarios en general, el IBPGR ha iniciado un proyecto piloto sobre redes globales por cultivo, en el cual se seleccionaron ocho cultivos contrastantes que representan a los siguientes grupos: cereales, fomjes, frutales, cultivos industriales, leguminosas, raíces y tubérculos, y hortalizas. Así, se podrá mejorar y coordinar las actividades de recolección, conservación, documentación y utilización de germoplasma de un cultivo en particular, eliminando la duplicación de esfuerzos a nivel nacional, regional o internacional.

\section{DOCUMENTACION Y LA UTILIZACION DE LOS RECURSOS FITOGENÉTICOS}

Con el objetivo de que la gran cantidad de información generada en un banco de germoplasma sea documentada eficientemente, se ha usado el sistema de bases de datos para la manipulación sistemática de la misma, debido a que tienen la flexibilidad de integrar de una forma ordenada los archivos de información correspondientes a las actividades de los programas de recursos genéticos. Estas, permiten el acceso y actualización de la información en forma sincronizada y consistente.

Una vez definidos los atributos de los archivos considerando la lista de descriptores, el sistema debe tener las características de ser compatible, flexible y adaptable, con el propósito de darle un mejor servicio principalmente a los programas nacionales de recursos genéticos, que generalmente tratan con diversos cultivos. Un sistema de bases de datos debe permitir introducir, actualizar y utilizar la información disponible sobre los datos de recolección y de pasaporte, caracterización y evaluación; el inventario actualizado del germoplasma disponible; la selección del material para regeneración ya sean introducciones recientes o incrementos, o bien material critico (tamaño de la muestra bajo conservación), porcentaje de germinación y contenido de humedad. Además, es ideal que el sistema/programa de documentación permita elaborar libros de campo, los cuales permitirán una más adecuada retroalimentación/actualización al sistema. También es deseable, que el programa permita al personal del banco, conducir análisis estadísticos (e.g. varianza, distribución de frecuencias, etc.) los cuales son de fundamental importancia en la evaluación, agrupación y selección del germoplasma. 


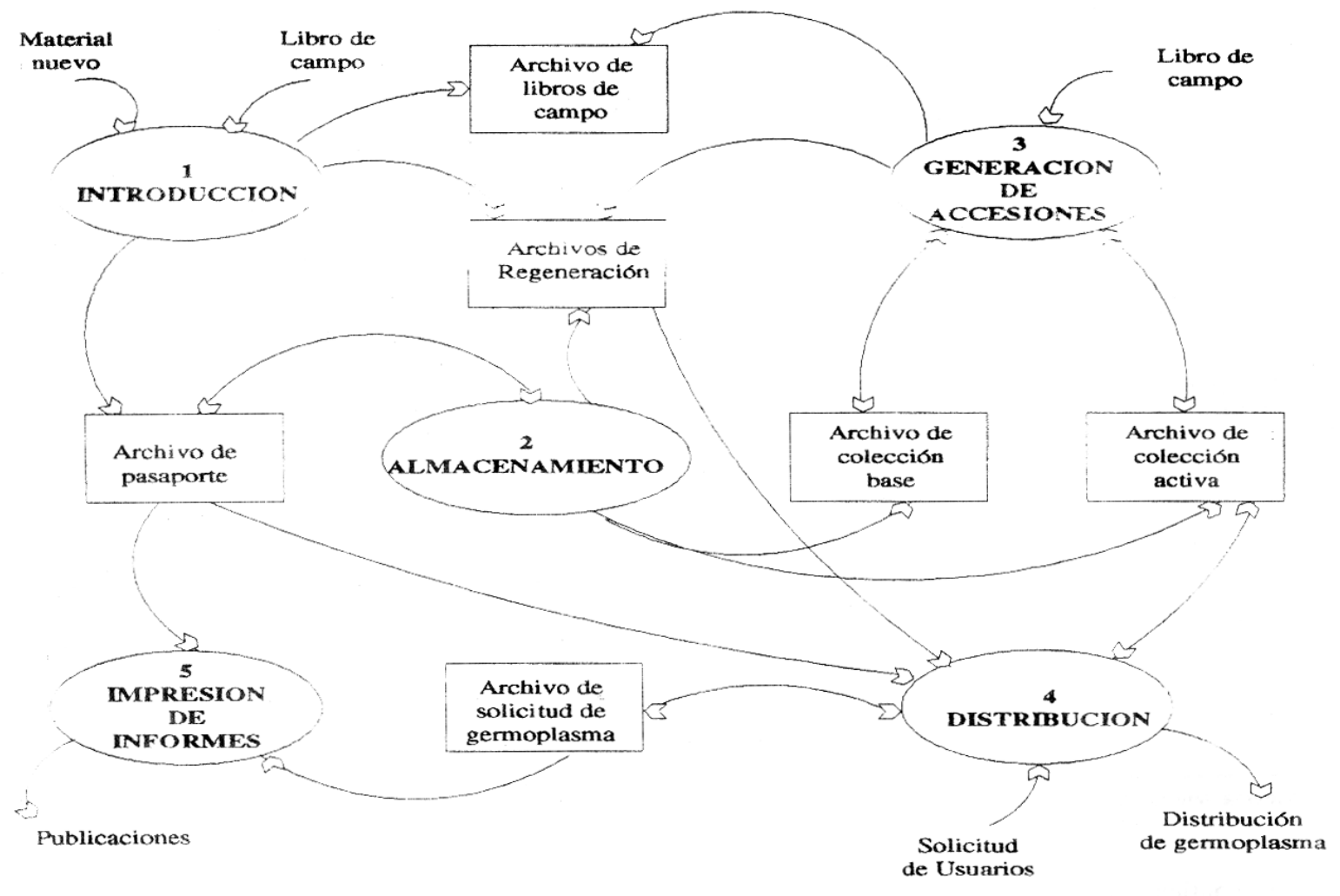

Figura 3 Interacción de actividades generales (círculo) y la generación de información (cuadro) en el Banco de Germoplasma de Maíz, CIMMYT

También es importante considerar la necesidad de procesar archivos de datos producidos por otros sistemas. De la misma manera, los archivos propios podrán ser intercambiados con sistemas de información de otros bancos de germoplasma. Eventualmente, el establecimiento de bases de datos regionales o mundiales estarán en la capacidad de identificar necesidades y prioridades en actividades de recolección, conservación, regeneración, etc., evitando en lo posible la duplicación de esfuerzos.

\section{BIBLIOGRAFIA}

CIMMYT 1989. Informe anual 1988. Centro Internacional de Mejoramiento de Maíz y Trigo. CIMMYT. México. D.F.

CIMMYT 1988. Recent advances in the conservation and utilization of genetic resources: Proceedings of the global maize germplasm workshop, CIMMYT. México, D. F.

DANTE, CJ. 1977. An Introduction to data base systems. Second edition. London U. K.
DANTE. CJ. 1987. Bases de datos. Una guía práctica. Traducción del Inglés por R. R. Ballester, Universidad Autónoma de Puebla. México.

FORD-LLOIYD, B. and M. Jackson 1986. Plant Genetic Resources. An introduction to their conservation and use. Edward Arnold Pub. London

HANDSON, J. 1985. Practical manuals for genebanks: No. 1. Procedures for handling seeds in genebanks. IBPGR, Rome.

IBPGR 1989. Annual report 1988, IBPGR. Rome.

KONOPKA. J.; HANSON, J. 1985. Documentation of genetic resources: Information handling systems for genebank rnanagement. IBPGR. Rome.

USDA 1985. Agriculture research service. Plant genctic and germplasm institute. A manual for public users of the germplasm resources information network. The GRIN databank. USDA-ARS. 